\title{
Partitioning of REE between calcite and carbonatitic melt containing P, S, Si at $900-650^{\circ} \mathrm{C}$ and $100 \mathrm{MPa}$
}

Dmitry A. Chebotarev ( $\sim$ ebotarev@gmail.com )

V.S. Sobolev Institute of Geology and Mineralogy SB RAS

Cora Wohlgemuth-Ueberwasser

Helmholtz Centre Potsdam - German Research Centre for Geosciences GFZ

\section{Research Article}

Keywords: carbonatites, rare earths, distribution coefficients, calcite

Posted Date: September 16th, 2021

DOI: https://doi.org/10.21203/rs.3.rs-885796/v1

License: (c) (1) This work is licensed under a Creative Commons Attribution 4.0 International License. Read Full License

Version of Record: A version of this preprint was published at Scientific Reports on February 28th, 2022. See the published version at https://doi.org/10.1038/s41598-022-07330-0. 


\section{Abstract}

Many carbonatites host deposits of REE and HFSE, and fractional crystallization might be a potentially powerful mechanism controlling magma enrichment by these metals to economically significant values. At present, information about the control of fractional crystallization by partition coefficients of oreforming elements at magmatic stage is incomplete. Here we present an experimental study of REE partitioning between carbonatite melt and calcite in the system $\mathrm{CaCO}_{3}-\mathrm{Na}_{2} \mathrm{CO}_{3}$ with varying amounts of $\mathrm{P}_{2} \mathrm{O}_{5}, \mathrm{~F}, \mathrm{Cl}, \mathrm{SiO}_{2}, \mathrm{SO}_{3}$ at $650-900^{\circ} \mathrm{C}$ and $100 \mathrm{MPa}$ using cold-seal pressure vessels and $\mathrm{LA}-\mathrm{ICP}-\mathrm{MS}$. The presence of phosphorus in the system generally increases the distribution coefficients but its effect decreases with increasing concentration. The influence of temperature is great: at $900-770^{\circ} \mathrm{C} D_{\mathrm{REE}} \geq 1$, while at lower temperatures the values are below unity. Silicon also promotes the fractionation of REE into calcite, while sulfur contributes to the retention of REE in the melt. Our results imply that calcite may impose significant control upon REE fractionation at the early stages of crystallization of carbonatite magma and can be a closest proxy for monitoring the REE content in initial melt.

\section{Introduction}

Carbonatites are rare carbonate-dominated mantle-derived rocks that, in some cases, host economically significant deposits of REE, Nb, Zr, which are important for modern high-tech and "green" industry. More than 527 carbonatite occurrences have been recorded in the world so far [1]. However, only about 30 mineralized carbonatites host economic resources of REE and $\mathrm{Nb}[2,3]$. The scientific and practical interest in carbonatites is great, but the processes of carbonatite formation and ore-grade enrichments of $\mathrm{Nb}$ and $\mathrm{REE}$ are far from being fully understood. Contemporary models of carbonatite petrogenesis fall into three major groups:

(1) primary origin of carbonatitic magmas by partial melting of a carbonated mantle source [4-15];

(2) derivative origin from an homogeneous alkaline silicate magma by silicate-carbonate liquid immiscibility [16, 17];

(3) derivative origin by extensive fractional crystallization of a carbonated silicate parental liquid [18, 19].

Identification of the exact process (or processes) responsible for the formation of carbonatitic magmas is hampered by widespread $\mathrm{Na}-\mathrm{K}$ metasomatism (fenitization) associated with carbonatites, which manifests the loss of alkalis and volatiles from their parental magmas [20]. In addition, many, if not most, intrusive carbonatites are cumulates, dominated by calcite or dolomite, differing in composition from their parental magma [21] and often affected by extensive textural and chemical re-equilibration after emplacement [22].

As seen from experimental works [14, 23-25], direct smelting from the mantle source and silicate-carbonate immiscibility are unable to provide concentration of REE to economically significant levels. Immiscibility experiments in various silicate-carbonate systems have demonstrated that $\mathrm{Pb}, \mathrm{Nb}$, Th, $\mathrm{U}$ and most of the REE preferentially partition into the silicate liquid, whereas $\mathrm{Sr}$, Ba and $\mathrm{F}$ partition into the conjugate liquid carbonate phase [23, 24, 26]. This pattern of element partitioning is inconsistent with a high content of primary LREE- and HFSE-rich minerals in carbonatites: fluorapatite, fluorcarbonates, monazite, pyrochlore, etc. Thus, another evolutionary mechanism is needed for the enrichment of carbonatitic magmas in REE and HFSE, and fractional crystallization might be powerful driver for it.

The majority of experimental estimates of the partition coefficients for REE have been obtained for the main rock-forming minerals (olivine, pyroxene, garnet, amphibole and biotite), and some accessory minerals (such as rutile, apatite, perovskite, and baddeleyite) at P-T conditions of the upper mantle [25, 27-37]. Experimental constraints on REE partitioning in carbonatitic magmas at crustal pressures are sparse. Because of low crystallization temperature and low viscosity of carbonatitic magmas, fractional crystallization can proceed in them up to shallow depths and control the distribution of REE and HFSE, leading to the formation of a deposit or a barren carbonatite body. Therefore, it is important to assess the effect of fractional crystallization at crustal conditions and to identify the influencing factors.

Carbonate minerals are the principal constituents of intrusive carbonatites: their content ranges from 50 modal \%, which is accepted as a nominal threshold for this rock type [38], to well over $90 \%$ in some varieties interpreted as cumulates [21]. Considering the high propensity of calcite to various postmagmatic changes, plasticity and recrystallization, observed trace element contents may be far from the original magmatic pattern. Hence, experimental modelling of trace element distribution between calcite and carbonatite melt at magmatic conditions is important.

Additional ligands such as $\mathrm{F}^{-}, \mathrm{OH}^{-}, \mathrm{Cl}^{-}, \mathrm{SO}_{4}{ }^{2-}$ and $\mathrm{PO}_{4}{ }^{3-}$ are likely to have strong effects upon the REE partitioning. In our previous work [39] we carried out a pilot series of experiments to obtain distribution coefficients for REE and HFSE between calcite, fluorite and nominally dry carbonatitic melts in synthetic compositions with $\mathrm{CaO}$ at 37-54 wt.\%, $\mathrm{Na}_{2} \mathrm{O} 7-24$ wt.\%, F 5-9 wt. $\%, \mathrm{P}_{2} \mathrm{O}_{5}$ between 0 and $9.5 \mathrm{wt} . \%$ at $650-900^{\circ} \mathrm{C}$ and $100 \mathrm{MPa}$. The distribution coefficients for individual REE in calcite in that study did not exceed 0.1 and in fluorite they were below 0.25 . Total REE concentrations in calcites were at about $500-700$ $\mathrm{ppm}$. Such concentrations are normal for primary igneous calcites in carbonatites [40]. However, there are described primary igneous calcites with REE contents at 1400-2000 ppm (e.g., the Aley complex, Canada [40]). This study was aimed at a more detailed assessment of the effects of melt composition, and especially the concentrations of $\mathrm{P}_{2} \mathrm{O}_{5}, \mathrm{SiO}_{2}$ and $\mathrm{SO}_{3}$ components on the REE distribution between calcite and melt at $900-650^{\circ} \mathrm{C}$ and $100 \mathrm{MPa}$.

\section{Results}

Carbonatite melts in all the experiments quenched as aggregates of fine-grained dendritic crystals with abundant fluid bubbles probably dominated by $\mathrm{CO}_{2}$ (Fig. 1, 2; Table 2, 3). In phosphate-bearing samples, quenched melt is full of needle-shaped crystals of quench Ca-phosphate, presumably apatite (Fig. 1a; 2a, b, c). In experiments with $\mathrm{SiO}_{2}$ fine crystals of wollastonite and Zr-Hf-silicate (zircon-hafnon) are also formed (Fig. 2d; Table 2). Oxides of HFSE and REE in all samples form aggregates of fine crystals with complex composition (baddeleyite, perovskite-lueshite solid solutions and crystals of pyrochlore supergroup) 
(Fig. 2a, b, c). The crystals are few microns in size and are not suitable for representative analyzes by microprobe or LA-ICP-MS. Rounded, irregularly shaped, drop-like fluorite crystals are formed in samples with high content of $F$ (Fig. 2a; Table 2).

Table 2

Experimental conditions and run products.

\begin{tabular}{|lllll|}
\hline Run \# & Mixture & Temperature, ${ }^{\circ} \mathrm{C}$ & Duration, hours & Phases \\
\hline 1 & NCFP-7 & 760 & 26 & Melt, fluid, calcite \\
\hline 2 & NCFP-8 & $900-850$ & 20 & Melt, fluid, calcite \\
\hline 3 & NCFP-9 & $900-650$ & 141 & Melt, fluid, calcite, fluorite, baddeleyite, pyrochlore \\
\hline 4 & NCP-1 & $900-830$ & 44 & Melt, calcite, baddeleyite \\
\hline 5 & NCPS-1 & $900-830$ & 25 & Melt, fluid, calcite \\
\hline 6 & NCP-1 & $820-770$ & 55 & Melt, fluid, calcite, REE and HFSE oxides \\
\hline 7 & NCPSi-1 & $820-770$ & 55 & Melt, fluid, calcite, wollastonite, zircon-hafnon \\
\hline 8 & NCPS-1 & $820-770$ & 55 & Melt, fluid, calcite, baddeleyite, pyrochlore \\
\hline 9 & NCP-2 & $820-770$ & 64 & Melt, fluid, calcite, Hf-oxide, REE and HFSE oxide \\
\hline 10 & NCPSi-2 & $820-770$ & 64 & Melt, fluid, calcite, wollastonite, zircon-hafnon \\
\hline 11 & NCPS-2 & $820-770$ & 64 & Melt, fluid, calcite, REE and HFSE oxides \\
\hline 12 & NCPSi-3 & $900-760$ & 52 & Melt, fluid, calcite, wollastonite \\
\hline
\end{tabular}


Table 3

Major composition of run products (in wt.\%).

\begin{tabular}{|c|c|c|c|c|c|c|c|c|c|c|c|}
\hline Run \# & Mixture & Phase & $\mathrm{Na}_{2} \mathrm{O}$ & $\mathrm{CaO}$ & $\mathrm{SiO}_{2}$ & $\mathrm{P}_{2} \mathrm{O}_{5}$ & $\mathrm{SO}_{3}$ & $\mathbf{F}$ & $\mathrm{Cl}$ & $\left(\mathrm{Cl}+\mathrm{F}_{2}\right)=-0$ & Total \\
\hline \multirow[t]{2}{*}{1} & \multirow[t]{2}{*}{ NCFP-1 } & Melt (3) & $12(2)$ & $48(2)$ & - & $12(3)$ & - & $9.7(9)$ & - & 4.10 & 77.00 \\
\hline & & Calcite (5) & $0.06(3)$ & $57(1)$ & - & - & - & - & - & - & 57.06 \\
\hline \multirow[t]{2}{*}{2} & \multirow[t]{2}{*}{ NCFP-3 } & Melt (6) & $6(1)$ & $54(1)$ & - & $11(2)$ & - & $11(1)$ & - & 4.50 & 77.00 \\
\hline & & Calcite (4) & 0.09 (3) & $56.1(0)$ & - & - & - & - & - & - & 56.20 \\
\hline \multirow[t]{2}{*}{3} & \multirow[t]{2}{*}{ NCFP-4 } & Melt (19) & $20(2)$ & $35.9(4)$ & - & $0.6(1)$ & - & $10.4(2)$ & - & - & 67.00 \\
\hline & & Calcite (16) & - & $54.5(3)$ & - & - & - & - & - & - & 54.60 \\
\hline \multirow[t]{3}{*}{4} & \multirow[t]{3}{*}{ NCP-1 } & Fluorite (17) & - & $71.6(4)$ & - & $1(1)$ & - & $48.4(7)$ & - & 20.40 & 100.00 \\
\hline & & Melt (9) & $20.5(17)$ & $35.9(4)$ & - & $0.6(1)$ & - & $10.4(2)$ & - & - & 67.40 \\
\hline & & Calcite (10) & - & $53.4(6)$ & - & - & - & - & - & - & 53.40 \\
\hline \multirow[t]{2}{*}{5} & \multirow[t]{2}{*}{ NCPS-1 } & Melt (12) & $20(2)$ & $35(2)$ & - & $6(4)$ & $0.8(3)$ & $0.63(9)$ & $0.3(2)$ & 0.40 & 62.86 \\
\hline & & Calcite (7) & - & $54(0)$ & - & - & - & - & - & - & 54.00 \\
\hline \multirow[t]{2}{*}{6} & \multirow[t]{2}{*}{ NCP-1 } & Melt (9) & $22.2(7)$ & $32(1)$ & - & $6(1)$ & - & $0.66(7)$ & $0.42(8)$ & 0.47 & 60.81 \\
\hline & & Calcite (11) & - & $54(0)$ & - & - & - & - & - & - & 54.00 \\
\hline \multirow[t]{2}{*}{7} & \multirow[t]{2}{*}{ NCPSi-1 } & Melt (2) & $23(2)$ & $31(2)$ & $2(3)$ & $2(2)$ & - & $0.6(4)$ & $0.4(1)$ & 0.43 & 58.82 \\
\hline & & Calcite (7) & - & $54(0)$ & - & - & - & - & - & - & 54.00 \\
\hline \multirow[t]{2}{*}{8} & \multirow[t]{2}{*}{ NCPS-1 } & Melt (17) & $23(2)$ & $31(2)$ & - & $4(4)$ & $1(0)$ & $0.66(8)$ & $0.17(2)$ & 0.36 & 59.92 \\
\hline & & Calcite (8) & - & $53.3(5)$ & - & - & - & - & - & - & 53.30 \\
\hline \multirow[t]{2}{*}{9} & \multirow[t]{2}{*}{ NCP-3 } & Melt (10) & $25.6(9)$ & $30.6(9)$ & - & $3(2)$ & - & - & $0.18(1)$ & 0.08 & 58.92 \\
\hline & & Calcite (8) & $0.4(3)$ & $54.7(2)$ & - & - & - & - & - & - & 55.10 \\
\hline \multirow[t]{2}{*}{10} & \multirow[t]{2}{*}{ NCPSi-3 } & Melt (11) & $21(3)$ & 30.7 (7) & $1.9(6)$ & - & - & - & $0.03(4)$ & 0.01 & 53.99 \\
\hline & & Calcite (4) & - & $54.6(3)$ & - & - & - & - & - & - & 54.60 \\
\hline \multirow[t]{2}{*}{11} & \multirow[t]{2}{*}{ NCPS-3 } & Melt (16) & $25(2)$ & $31(2)$ & - & $3(4)$ & $0.7(1)$ & $0.45(1)$ & $0.07(2)$ & 0.22 & 58.97 \\
\hline & & Calcite (9) & $0.27(6)$ & $54.6(7)$ & - & - & - & - & - & - & 54.80 \\
\hline \multirow[t]{3}{*}{12} & \multirow[t]{3}{*}{ NCPSi-4 } & & & & & & & & & & \\
\hline & & Melt (8) & $26(2)$ & 29.8 (4) & - & $2(3)$ & - & - & $0.06(0)$ & 0.03 & 56.97 \\
\hline & & Calcite (6) & - & $53.7(4)$ & - & - & - & - & - & - & 53.70 \\
\hline
\end{tabular}

Calcite has various morphology: rhombohedral or prismatic (Fig. 1a; 2a), amorphous (Fig. 1b), needle-like (Fig. 1d, f) and tabular rounded crystals (laths) up to 250 micrometers in most experiments (Fig. 1c, e; 2 c), which are typical for early generations of calcite in carbonatites [44]. The amount of calcite crystals in some samples is so high that they form a crystal mush at the bottom of the samples (Fig. 1c, e). Calcite major element composition is close to that of stoichiometric Ca carbonate (Table 3).

Trace elements compoisition of the run products given in Table 4. Partition coefficients calculated according to the Nernst formula as mass ratios of element content in a crystalline phase to its content in the melt: $D_{\text {Element }}=C^{\text {Crystal } / C^{\text {Melt }}}$ (Table 5). $D_{S r}$ is $>1$ for all samples (Fig. 3). In all experiments $D_{S r}>>D_{R E E}$, exept NCPSi mixtures. Almost all $D_{\text {REE }}$ plots show a positive Eu and $Y$ anomalies. In general, the slope of the $D_{\text {REE }}$ plots is positive, with the exception of NCPSi samples, what is apparently associated with active fractionation of HREE into wollastonite. 
Table 4

Trace element composition of run products (in ppm).

\begin{tabular}{|c|c|c|c|c|c|c|c|c|c|c|c|c|c|c|c|c|c|c|}
\hline $\begin{array}{l}\text { Run } \\
\#\end{array}$ & Mixture & Phase & $\mathrm{Sr}$ & La & $\mathrm{Ce}$ & $\operatorname{Pr}$ & Nd & $\mathrm{Sm}$ & Eu & Gd & $\mathrm{Tb}$ & Dy & Ho & $Y$ & $\mathrm{Er}$ & $\mathrm{Tm}$ & $\mathrm{Yb}$ & Lu \\
\hline \multirow[t]{2}{*}{1} & NCFP-1 & Melt (5) & $\begin{array}{l}450 \\
(24)\end{array}$ & $\begin{array}{l}183 \\
(10)\end{array}$ & $\begin{array}{l}155 \\
(9)\end{array}$ & $\begin{array}{l}160 \\
(10)\end{array}$ & $\begin{array}{l}153 \\
(8)\end{array}$ & $\begin{array}{l}178 \\
(7)\end{array}$ & $\begin{array}{l}155 \\
(8)\end{array}$ & $\begin{array}{l}161 \\
(10)\end{array}$ & $\begin{array}{l}155 \\
(7)\end{array}$ & $\begin{array}{l}165 \\
(11)\end{array}$ & $\begin{array}{l}173 \\
(8)\end{array}$ & $\begin{array}{l}156 \\
(8)\end{array}$ & $\begin{array}{l}172 \\
(7)\end{array}$ & $\begin{array}{l}172 \\
(7)\end{array}$ & $\begin{array}{l}177 \\
(10)\end{array}$ & $\begin{array}{l}163 \\
(11)\end{array}$ \\
\hline & & Calcite (5) & $\begin{array}{l}741 \\
(24)\end{array}$ & $\begin{array}{l}95 \\
(10)\end{array}$ & $\begin{array}{l}94 \\
(9)\end{array}$ & $\begin{array}{l}121 \\
(10)\end{array}$ & $\begin{array}{l}118 \\
(8)\end{array}$ & $\begin{array}{l}144 \\
(7)\end{array}$ & $\begin{array}{l}145 \\
(8)\end{array}$ & $\begin{array}{l}138 \\
(10)\end{array}$ & $\begin{array}{l}138 \\
(7)\end{array}$ & $\begin{array}{l}160 \\
(11)\end{array}$ & $\begin{array}{l}166 \\
(8)\end{array}$ & $\begin{array}{l}122 \\
(8)\end{array}$ & $\begin{array}{l}174 \\
(7)\end{array}$ & $\begin{array}{l}184 \\
(7)\end{array}$ & $\begin{array}{l}208 \\
(10)\end{array}$ & $\begin{array}{l}192 \\
(11)\end{array}$ \\
\hline \multirow[t]{2}{*}{2} & NCFP-3 & Melt (5) & $\begin{array}{l}426 \\
(9)\end{array}$ & $\begin{array}{l}132 \\
(4)\end{array}$ & $\begin{array}{l}120 \\
(6)\end{array}$ & $\begin{array}{l}137 \\
(10)\end{array}$ & $\begin{array}{l}126 \\
(6)\end{array}$ & $\begin{array}{l}134 \\
(7)\end{array}$ & $\begin{array}{l}135 \\
(4)\end{array}$ & $\begin{array}{l}132 \\
(7)\end{array}$ & $\begin{array}{l}126 \\
(5)\end{array}$ & $\begin{array}{l}135 \\
(8)\end{array}$ & $\begin{array}{l}135 \\
(5)\end{array}$ & $\begin{array}{l}129 \\
(2)\end{array}$ & $\begin{array}{l}136 \\
(7)\end{array}$ & $\begin{array}{l}138 \\
(3)\end{array}$ & $\begin{array}{l}175 \\
(12)\end{array}$ & $\begin{array}{l}128 \\
(3)\end{array}$ \\
\hline & & Calcite (3) & $\begin{array}{l}708 \\
(6)\end{array}$ & $\begin{array}{l}103 \\
(5)\end{array}$ & $\begin{array}{l}101 \\
(6)\end{array}$ & $\begin{array}{l}122 \\
(8)\end{array}$ & $\begin{array}{l}121 \\
(9)\end{array}$ & $\begin{array}{l}143 \\
(7)\end{array}$ & $\begin{array}{l}181 \\
(6)\end{array}$ & $\begin{array}{l}142 \\
(7)\end{array}$ & $\begin{array}{l}143 \\
(9)\end{array}$ & $\begin{array}{l}157 \\
(11)\end{array}$ & $\begin{array}{l}161 \\
(8)\end{array}$ & $\begin{array}{l}129 \\
(2)\end{array}$ & $\begin{array}{l}168 \\
(10)\end{array}$ & $\begin{array}{l}176 \\
(12)\end{array}$ & $\begin{array}{l}215 \\
(10)\end{array}$ & $\begin{array}{l}178 \\
(2)\end{array}$ \\
\hline \multirow[t]{4}{*}{3} & NCFP-4 & Melt (5) & $\begin{array}{l}703 \\
(60)\end{array}$ & $\begin{array}{l}618 \\
(51)\end{array}$ & $\begin{array}{l}389 \\
(13)\end{array}$ & $\begin{array}{l}427 \\
(13)\end{array}$ & $\begin{array}{l}444 \\
(29)\end{array}$ & $\begin{array}{l}435 \\
(34)\end{array}$ & $\begin{array}{l}499 \\
(40)\end{array}$ & $\begin{array}{l}453 \\
(34)\end{array}$ & $\begin{array}{l}438 \\
(27)\end{array}$ & $\begin{array}{l}438 \\
(20)\end{array}$ & $\begin{array}{l}665 \\
(62)\end{array}$ & $\begin{array}{l}474 \\
(43)\end{array}$ & $\begin{array}{l}443 \\
(11)\end{array}$ & $\begin{array}{l}471 \\
(14)\end{array}$ & $\begin{array}{l}535 \\
(24)\end{array}$ & $\begin{array}{l}552 \\
(34)\end{array}$ \\
\hline & & Calcite (5) & $\begin{array}{l}520 \\
(73)\end{array}$ & $\begin{array}{l}85 \\
(44)\end{array}$ & $\begin{array}{l}79 \\
(38)\end{array}$ & $\begin{array}{l}90 \\
(42)\end{array}$ & $\begin{array}{l}95 \\
(44)\end{array}$ & $\begin{array}{l}95 \\
(37)\end{array}$ & $\begin{array}{l}123 \\
(43)\end{array}$ & $\begin{array}{l}96 \\
(37)\end{array}$ & $\begin{array}{l}97 \\
(32)\end{array}$ & $\begin{array}{l}102 \\
(30)\end{array}$ & $\begin{array}{l}110 \\
(29)\end{array}$ & $\begin{array}{l}83 \\
(20)\end{array}$ & $\begin{array}{l}110 \\
(28)\end{array}$ & $\begin{array}{l}115 \\
(27)\end{array}$ & $\begin{array}{l}119 \\
(25)\end{array}$ & $\begin{array}{l}123 \\
(22)\end{array}$ \\
\hline & & $\begin{array}{l}\text { Fluorite } \\
\text { (3) }\end{array}$ & $\begin{array}{l}195 \\
(47)\end{array}$ & bdl & bdl & $\begin{array}{l}11 \\
(11)\end{array}$ & $\begin{array}{l}9 \\
(3)\end{array}$ & $\begin{array}{l}29 \\
(11)\end{array}$ & $\begin{array}{l}33 \\
(1)\end{array}$ & $\begin{array}{l}55 \\
(25)\end{array}$ & $\begin{array}{l}51 \\
(22)\end{array}$ & $\begin{array}{l}56 \\
(22)\end{array}$ & $\begin{array}{l}109 \\
(79)\end{array}$ & $\begin{array}{l}152 \\
(94)\end{array}$ & $\begin{array}{l}46 \\
(26)\end{array}$ & $\begin{array}{l}85 \\
(65)\end{array}$ & $\begin{array}{l}48 \\
(13)\end{array}$ & $\begin{array}{l}55 \\
(24)\end{array}$ \\
\hline & & $\begin{array}{l}\text { Ca- } \\
\text { phosphate } \\
\text { (1) }\end{array}$ & 581 & 116 & 103 & 114 & 116 & 118 & 154 & 129 & 128 & 132 & 141 & 85 & 134 & 136 & 139 & 134 \\
\hline \multirow[t]{2}{*}{4} & NCP-1 & Melt (9) & $\begin{array}{l}126 \\
(18)\end{array}$ & $\begin{array}{l}274 \\
(29)\end{array}$ & $\begin{array}{l}225 \\
(29)\end{array}$ & $\begin{array}{l}261 \\
(54)\end{array}$ & $\begin{array}{l}285 \\
(59)\end{array}$ & $\begin{array}{l}282 \\
(52)\end{array}$ & $\begin{array}{l}289 \\
(48)\end{array}$ & $\begin{array}{l}268 \\
(47)\end{array}$ & $\begin{array}{l}258 \\
(51)\end{array}$ & $\begin{array}{l}247 \\
(51)\end{array}$ & $\begin{array}{l}253 \\
(35)\end{array}$ & $\begin{array}{l}268 \\
(41)\end{array}$ & $\begin{array}{l}260 \\
(11)\end{array}$ & $\begin{array}{l}286 \\
(47)\end{array}$ & $\begin{array}{l}308 \\
(63)\end{array}$ & $\begin{array}{l}314 \\
(62)\end{array}$ \\
\hline & & Calcite (5) & $\begin{array}{l}150 \\
(28)\end{array}$ & $\begin{array}{l}248 \\
(40)\end{array}$ & $\begin{array}{l}196 \\
(22)\end{array}$ & $\begin{array}{l}232 \\
(10)\end{array}$ & $\begin{array}{l}265 \\
(25)\end{array}$ & $\begin{array}{l}279 \\
(34)\end{array}$ & $\begin{array}{l}292 \\
(56)\end{array}$ & $\begin{array}{l}296 \\
(57)\end{array}$ & $\begin{array}{l}277 \\
(52)\end{array}$ & $\begin{array}{l}270 \\
(48)\end{array}$ & $\begin{array}{l}292 \\
(52)\end{array}$ & $\begin{array}{l}281 \\
(32)\end{array}$ & $\begin{array}{l}303 \\
(64)\end{array}$ & $\begin{array}{l}336 \\
(84)\end{array}$ & $\begin{array}{l}318 \\
(32)\end{array}$ & $\begin{array}{l}343 \\
(31)\end{array}$ \\
\hline \multirow[t]{2}{*}{5} & $\begin{array}{l}\text { NCPS- } \\
1\end{array}$ & Melt (5) & $\begin{array}{l}80 \\
(3)\end{array}$ & $\begin{array}{l}169 \\
(6)\end{array}$ & $\begin{array}{l}161 \\
(8)\end{array}$ & $\begin{array}{l}179 \\
(8)\end{array}$ & $\begin{array}{l}191 \\
(6)\end{array}$ & $\begin{array}{l}204 \\
(10)\end{array}$ & $\begin{array}{l}197 \\
(9)\end{array}$ & $\begin{array}{l}201 \\
(9)\end{array}$ & $\begin{array}{l}196 \\
(8)\end{array}$ & $\begin{array}{l}193 \\
(9)\end{array}$ & $\begin{array}{l}188 \\
(9)\end{array}$ & $\begin{array}{l}176 \\
(10)\end{array}$ & $\begin{array}{l}183 \\
(9)\end{array}$ & $\begin{array}{l}182 \\
(11)\end{array}$ & $\begin{array}{l}186 \\
(14)\end{array}$ & $\begin{array}{l}182 \\
(12)\end{array}$ \\
\hline & & Calcite (5) & $\begin{array}{l}180 \\
(21)\end{array}$ & $\begin{array}{l}262 \\
(42)\end{array}$ & $\begin{array}{l}214 \\
(39)\end{array}$ & $\begin{array}{l}233 \\
(30)\end{array}$ & $\begin{array}{l}238 \\
(27)\end{array}$ & $\begin{array}{l}226 \\
(26)\end{array}$ & $\begin{array}{l}264 \\
(38)\end{array}$ & $\begin{array}{l}216 \\
(29)\end{array}$ & $\begin{array}{l}203 \\
(21)\end{array}$ & $\begin{array}{l}207 \\
(28)\end{array}$ & $\begin{array}{l}193 \\
(19)\end{array}$ & $\begin{array}{l}190 \\
(22)\end{array}$ & $\begin{array}{l}189 \\
(24)\end{array}$ & $\begin{array}{l}185 \\
(20)\end{array}$ & $\begin{array}{l}193 \\
(30)\end{array}$ & $\begin{array}{l}200 \\
(27)\end{array}$ \\
\hline \multirow[t]{2}{*}{6} & NCP-1 & Melt (5) & $\begin{array}{l}109 \\
(4)\end{array}$ & $\begin{array}{l}268 \\
(19)\end{array}$ & $\begin{array}{l}219 \\
(14)\end{array}$ & $\begin{array}{l}244 \\
(16)\end{array}$ & $\begin{array}{l}257 \\
(14)\end{array}$ & $\begin{array}{l}262 \\
(13)\end{array}$ & $\begin{array}{l}263 \\
(14)\end{array}$ & $\begin{array}{l}265 \\
(18)\end{array}$ & $\begin{array}{l}260 \\
(15)\end{array}$ & $\begin{array}{l}262 \\
(14)\end{array}$ & $\begin{array}{l}262 \\
(15)\end{array}$ & $\begin{array}{l}253 \\
(15)\end{array}$ & $\begin{array}{l}252 \\
(13)\end{array}$ & $\begin{array}{l}256 \\
(16)\end{array}$ & $\begin{array}{l}275 \\
(21)\end{array}$ & $\begin{array}{l}277 \\
(18)\end{array}$ \\
\hline & & Calcite (5) & $\begin{array}{l}137 \\
(6)\end{array}$ & $\begin{array}{l}147 \\
(28)\end{array}$ & $\begin{array}{l}132 \\
(28)\end{array}$ & $\begin{array}{l}148 \\
(32)\end{array}$ & $\begin{array}{l}166 \\
(33)\end{array}$ & $\begin{array}{l}172 \\
(42)\end{array}$ & $\begin{array}{l}196 \\
(36)\end{array}$ & $\begin{array}{l}185 \\
(46)\end{array}$ & $\begin{array}{l}181 \\
(49)\end{array}$ & $\begin{array}{l}184 \\
(51)\end{array}$ & $\begin{array}{l}182 \\
(48)\end{array}$ & $\begin{array}{l}184 \\
(45)\end{array}$ & $\begin{array}{l}182 \\
(50)\end{array}$ & $\begin{array}{l}186 \\
(54)\end{array}$ & $\begin{array}{l}205 \\
(70)\end{array}$ & $\begin{array}{l}211 \\
(62)\end{array}$ \\
\hline \multirow[t]{2}{*}{7} & $\begin{array}{l}\text { NCPSi- } \\
1\end{array}$ & Melt (3) & $\begin{array}{l}124 \\
(15)\end{array}$ & $\begin{array}{l}210 \\
(47)\end{array}$ & $\begin{array}{l}167 \\
(24)\end{array}$ & $\begin{array}{l}202 \\
(28)\end{array}$ & $\begin{array}{l}221 \\
(17)\end{array}$ & $\begin{array}{l}240 \\
(56)\end{array}$ & $\begin{array}{l}234 \\
(28)\end{array}$ & $\begin{array}{l}243 \\
(26)\end{array}$ & $\begin{array}{l}224 \\
(32)\end{array}$ & $\begin{array}{l}212 \\
(27)\end{array}$ & $\begin{array}{l}225 \\
(40)\end{array}$ & $\begin{array}{l}222 \\
(38)\end{array}$ & $\begin{array}{l}226 \\
(37)\end{array}$ & $\begin{array}{l}235 \\
(45)\end{array}$ & $\begin{array}{l}266 \\
(62)\end{array}$ & $\begin{array}{l}272 \\
(62)\end{array}$ \\
\hline & & Calcite (3) & $\begin{array}{l}156 \\
(4)\end{array}$ & $\begin{array}{l}125 \\
(2)\end{array}$ & $\begin{array}{l}84 \\
(1)\end{array}$ & $\begin{array}{l}86 \\
(1)\end{array}$ & $\begin{array}{l}82 \\
(1)\end{array}$ & $\begin{array}{l}71 \\
(1)\end{array}$ & $\begin{array}{l}78 \\
(2)\end{array}$ & $\begin{array}{l}70 \\
(2)\end{array}$ & $\begin{array}{l}65 \\
(0)\end{array}$ & $\begin{array}{l}66 \\
(1)\end{array}$ & $\begin{array}{l}69 \\
(2)\end{array}$ & $\begin{array}{l}72 \\
(1)\end{array}$ & $\begin{array}{l}65 \\
(0)\end{array}$ & $\begin{array}{l}64 \\
(1)\end{array}$ & $\begin{array}{l}65 \\
(1)\end{array}$ & $\begin{array}{l}67 \\
(1)\end{array}$ \\
\hline \multirow[t]{2}{*}{8} & $\begin{array}{l}\text { NCPS- } \\
1\end{array}$ & Melt (5) & $\begin{array}{l}87 \\
(2)\end{array}$ & $\begin{array}{l}204 \\
(11)\end{array}$ & $\begin{array}{l}178 \\
(10)\end{array}$ & $\begin{array}{l}199 \\
(9)\end{array}$ & $\begin{array}{l}208 \\
(7)\end{array}$ & $\begin{array}{l}220 \\
(10)\end{array}$ & $\begin{array}{l}223 \\
(9)\end{array}$ & $\begin{array}{l}222 \\
(8)\end{array}$ & $\begin{array}{l}218 \\
(10)\end{array}$ & $\begin{array}{l}215 \\
(10)\end{array}$ & $\begin{array}{l}219 \\
(11)\end{array}$ & $\begin{array}{l}201 \\
(12)\end{array}$ & $\begin{array}{l}208 \\
(13)\end{array}$ & $\begin{array}{l}206 \\
(17)\end{array}$ & $\begin{array}{l}214 \\
(21)\end{array}$ & $\begin{array}{l}219 \\
(26)\end{array}$ \\
\hline & & Calcite (5) & $\begin{array}{l}119 \\
(8)\end{array}$ & $\begin{array}{l}147 \\
(14)\end{array}$ & $\begin{array}{l}122 \\
(12)\end{array}$ & $\begin{array}{l}139 \\
(12)\end{array}$ & $\begin{array}{l}153 \\
(15)\end{array}$ & $\begin{array}{l}155 \\
(15)\end{array}$ & $\begin{array}{l}173 \\
(13)\end{array}$ & $\begin{array}{l}167 \\
(17)\end{array}$ & $\begin{array}{l}163 \\
(17)\end{array}$ & $\begin{array}{l}164 \\
(16)\end{array}$ & $\begin{array}{l}176 \\
(17)\end{array}$ & $\begin{array}{l}166 \\
(15)\end{array}$ & $\begin{array}{l}167 \\
(17)\end{array}$ & $\begin{array}{l}167 \\
(15)\end{array}$ & $\begin{array}{l}175 \\
(17)\end{array}$ & $\begin{array}{l}183 \\
(18)\end{array}$ \\
\hline \multirow[t]{2}{*}{9} & NCP-3 & Melt (5) & $\begin{array}{l}67 \\
(8)\end{array}$ & $\begin{array}{l}27 \\
(6)\end{array}$ & $\begin{array}{l}21 \\
(4)\end{array}$ & $\begin{array}{l}24 \\
(4)\end{array}$ & $\begin{array}{l}27 \\
(4)\end{array}$ & $\begin{array}{l}29 \\
(4)\end{array}$ & $\begin{array}{l}28 \\
(4)\end{array}$ & $\begin{array}{l}27 \\
(4)\end{array}$ & $\begin{array}{l}26 \\
(6)\end{array}$ & $\begin{array}{l}26 \\
(6)\end{array}$ & $\begin{array}{l}25 \\
(5)\end{array}$ & $\begin{array}{l}23 \\
(5)\end{array}$ & $\begin{array}{l}23 \\
(5)\end{array}$ & $\begin{array}{l}23 \\
(5)\end{array}$ & $\begin{array}{l}24 \\
(5)\end{array}$ & $\begin{array}{l}24 \\
(5)\end{array}$ \\
\hline & & Calcite (5) & $\begin{array}{l}78 \\
(8)\end{array}$ & $\begin{array}{l}24 \\
(6)\end{array}$ & $\begin{array}{l}20 \\
(4)\end{array}$ & $\begin{array}{l}22 \\
(5)\end{array}$ & $\begin{array}{l}27 \\
(6)\end{array}$ & $\begin{array}{l}29 \\
(6)\end{array}$ & $\begin{array}{l}31 \\
(6)\end{array}$ & $\begin{array}{l}29 \\
(6)\end{array}$ & $\begin{array}{l}26 \\
(5)\end{array}$ & $\begin{array}{l}26 \\
(5)\end{array}$ & $\begin{array}{l}26 \\
(5)\end{array}$ & $\begin{array}{l}25 \\
(6)\end{array}$ & $\begin{array}{l}23 \\
(4)\end{array}$ & $\begin{array}{l}24 \\
(4)\end{array}$ & $\begin{array}{l}25 \\
(5)\end{array}$ & $\begin{array}{l}26 \\
(6)\end{array}$ \\
\hline \multirow[t]{2}{*}{10} & $\begin{array}{l}\text { NCPSi- } \\
3\end{array}$ & Melt (5) & $\begin{array}{l}57 \\
(6)\end{array}$ & $\begin{array}{l}17 \\
(2)\end{array}$ & $\begin{array}{l}14 \\
(1)\end{array}$ & $\begin{array}{l}16 \\
(0)\end{array}$ & $\begin{array}{l}17 \\
(0)\end{array}$ & $\begin{array}{l}17 \\
(2)\end{array}$ & $\begin{array}{l}21 \\
(4)\end{array}$ & $\begin{array}{l}21 \\
(7)\end{array}$ & $\begin{array}{l}21 \\
(9)\end{array}$ & $\begin{array}{l}20 \\
(12)\end{array}$ & $\begin{array}{l}21 \\
(13)\end{array}$ & $\begin{array}{l}24 \\
(15)\end{array}$ & $\begin{array}{l}20 \\
(14)\end{array}$ & $\begin{array}{l}21 \\
(16)\end{array}$ & $\begin{array}{l}21 \\
(17)\end{array}$ & $\begin{array}{l}26 \\
(26)\end{array}$ \\
\hline & & Calcite (4) & $\begin{array}{l}136 \\
(14)\end{array}$ & $\begin{array}{l}35 \\
(2)\end{array}$ & $\begin{array}{l}26 \\
(0)\end{array}$ & $\begin{array}{l}28 \\
(0)\end{array}$ & $\begin{array}{l}27 \\
(1)\end{array}$ & $\begin{array}{l}27 \\
(1)\end{array}$ & $\begin{array}{l}31 \\
(1)\end{array}$ & $\begin{array}{l}28 \\
(0)\end{array}$ & $\begin{array}{l}25 \\
(1)\end{array}$ & $\begin{array}{l}21 \\
(0)\end{array}$ & $\begin{array}{l}20 \\
(1)\end{array}$ & $\begin{array}{l}25 \\
(2)\end{array}$ & $\begin{array}{l}17 \\
(1)\end{array}$ & $\begin{array}{l}15 \\
(1)\end{array}$ & $\begin{array}{l}15 \\
(2)\end{array}$ & $\begin{array}{l}15 \\
(2)\end{array}$ \\
\hline \multirow[t]{2}{*}{11} & $\begin{array}{l}\text { NCPS- } \\
3\end{array}$ & Melt (5) & $\begin{array}{l}69 \\
(1)\end{array}$ & $\begin{array}{l}27 \\
(2)\end{array}$ & $\begin{array}{l}20 \\
(1)\end{array}$ & $\begin{array}{l}24 \\
(1)\end{array}$ & $\begin{array}{l}27 \\
(1)\end{array}$ & $\begin{array}{l}27 \\
(1)\end{array}$ & $\begin{array}{l}27 \\
(1)\end{array}$ & $\begin{array}{l}26 \\
(1)\end{array}$ & $\begin{array}{l}27 \\
(1)\end{array}$ & $\begin{array}{l}26 \\
(1)\end{array}$ & $\begin{array}{l}27 \\
(1)\end{array}$ & $\begin{array}{l}25 \\
(1)\end{array}$ & $\begin{array}{l}25 \\
(1)\end{array}$ & $\begin{array}{l}24 \\
(1)\end{array}$ & $\begin{array}{l}26 \\
(1)\end{array}$ & $\begin{array}{l}26 \\
(0)\end{array}$ \\
\hline & & Calcite (5) & $\begin{array}{l}89 \\
(18)\end{array}$ & $\begin{array}{l}29 \\
(12)\end{array}$ & $\begin{array}{l}23 \\
(9)\end{array}$ & $\begin{array}{l}27 \\
(12)\end{array}$ & $\begin{array}{l}31 \\
(14)\end{array}$ & $\begin{array}{l}33 \\
(14)\end{array}$ & $\begin{array}{l}33 \\
(11)\end{array}$ & $\begin{array}{l}34 \\
(15)\end{array}$ & $\begin{array}{l}32 \\
(12)\end{array}$ & $\begin{array}{l}32 \\
(11)\end{array}$ & $\begin{array}{l}33 \\
(11)\end{array}$ & $\begin{array}{l}30 \\
(8)\end{array}$ & $\begin{array}{l}31 \\
(10)\end{array}$ & $\begin{array}{l}28 \\
(8)\end{array}$ & $\begin{array}{l}32 \\
(10)\end{array}$ & $\begin{array}{l}33 \\
(8)\end{array}$ \\
\hline \multirow[t]{2}{*}{12} & $\begin{array}{l}\text { NCPSi- } \\
4\end{array}$ & Melt (5) & $\begin{array}{l}46 \\
(2)\end{array}$ & $\begin{array}{l}107 \\
(8)\end{array}$ & $\begin{array}{l}92 \\
(6)\end{array}$ & $\begin{array}{l}106 \\
(8)\end{array}$ & $\begin{array}{l}116 \\
(8)\end{array}$ & $\begin{array}{l}117 \\
(13)\end{array}$ & $\begin{array}{l}136 \\
(10)\end{array}$ & $\begin{array}{l}112 \\
(18)\end{array}$ & $\begin{array}{l}105 \\
(19)\end{array}$ & $\begin{array}{l}96 \\
(19)\end{array}$ & $\begin{array}{l}95 \\
(24)\end{array}$ & $\begin{array}{l}87 \\
(22)\end{array}$ & $\begin{array}{l}83 \\
(26)\end{array}$ & $\begin{array}{l}81 \\
(32)\end{array}$ & $\begin{array}{l}77 \\
(32)\end{array}$ & $\begin{array}{l}73 \\
(37)\end{array}$ \\
\hline & & Calcite (5) & $\begin{array}{l}73 \\
(5)\end{array}$ & $\begin{array}{l}200 \\
(17)\end{array}$ & $\begin{array}{l}154 \\
(12)\end{array}$ & $\begin{array}{l}163 \\
(12)\end{array}$ & $\begin{array}{l}169 \\
(10)\end{array}$ & $\begin{array}{l}152 \\
(7)\end{array}$ & $\begin{array}{l}168 \\
(12)\end{array}$ & $\begin{array}{l}150 \\
(10)\end{array}$ & $\begin{array}{l}138 \\
(6)\end{array}$ & $\begin{array}{l}132 \\
(6)\end{array}$ & $\begin{array}{l}129 \\
(5)\end{array}$ & $\begin{array}{l}126 \\
(2)\end{array}$ & $\begin{array}{l}114 \\
(5)\end{array}$ & $\begin{array}{l}111 \\
(5)\end{array}$ & $\begin{array}{l}110 \\
(5)\end{array}$ & $\begin{array}{l}108 \\
(6)\end{array}$ \\
\hline
\end{tabular}

The numbers in the parentheses next to the phase name indicates the number of analyzes. The numbers in the parentheses next to the analyzes are the standard deviation and reported as the least unit cited. For example, 182 (48) should be read as $182 \pm 48 \mathrm{ppm}$. 
Table 5

Calcite-melt partition coefficients of trace elements.

\begin{tabular}{|c|c|c|c|c|c|c|c|c|c|c|c|c|c|c|c|c|c|c|c|}
\hline \multirow[t]{3}{*}{ Element } & \multicolumn{2}{|l|}{1} & \multicolumn{2}{|l|}{2} & \multicolumn{2}{|l|}{3} & \multicolumn{2}{|l|}{4} & \multicolumn{2}{|l|}{5} & \multicolumn{2}{|l|}{6} & \multicolumn{2}{|l|}{7} & \multicolumn{2}{|l|}{8} & \multicolumn{2}{|l|}{9} & \multirow{2}{*}{$\frac{10}{\mathrm{NC}}$} \\
\hline & \multicolumn{2}{|c|}{ NCFP-7 } & \multicolumn{2}{|c|}{ NCFP-8 } & \multicolumn{2}{|c|}{ NCFP-9 } & \multicolumn{2}{|c|}{ NCP-1 } & \multicolumn{2}{|c|}{ NCPS-1 } & \multicolumn{2}{|c|}{ NCP-1 } & \multicolumn{2}{|c|}{ NCPSi-1 } & \multicolumn{2}{|c|}{ NCPS-1 } & \multicolumn{2}{|c|}{ NCP-2 } & \\
\hline & $D_{\text {REE }}$ & $\sigma$ & $D_{\text {REE }}$ & $\sigma$ & $\mathrm{D}_{\mathrm{REE}}$ & $\sigma$ & $\mathrm{D}_{\mathrm{REE}}$ & $\sigma$ & $D_{\text {REE }}$ & $\sigma$ & $D_{\text {REE }}$ & $\sigma$ & $\mathrm{D}_{\mathrm{REE}}$ & $\sigma$ & $\mathrm{D}_{\mathrm{REE}}$ & $\sigma$ & $\mathrm{D}_{\mathrm{REE}}$ & $\sigma$ & $\mathrm{D}_{\mathrm{RI}}$ \\
\hline $\mathrm{Sr}$ & 1.65 & 0.07 & 1.66 & 0.04 & 0.77 & 0.04 & 1.25 & 0.15 & 2.42 & 0.11 & 1.23 & 0.05 & 2.33 & 0.30 & 1.37 & 0.12 & 1.16 & 0.19 & 2.3 \\
\hline La & 0.52 & 0.11 & 0.78 & 0.04 & 0.09 & 0.01 & 0.79 & 0.08 & 1.72 & 0.10 & 0.55 & 0.05 & 4.70 & 1.13 & 0.71 & 0.07 & 0.90 & 0.32 & 2.1 \\
\hline $\mathrm{Ce}$ & 0.61 & 0.11 & 0.84 & 0.06 & 0.14 & 0.01 & 0.89 & 0.07 & 1.49 & 0.08 & 0.60 & 0.04 & 3.95 & 0.81 & 0.67 & 0.07 & 0.92 & 0.26 & 1.8 \\
\hline $\mathrm{Pr}$ & 0.76 & 0.09 & 0.89 & 0.09 & 0.15 & 0.01 & 1.00 & 0.08 & 1.42 & 0.09 & 0.61 & 0.05 & 3.64 & 0.65 & 0.69 & 0.08 & 0.94 & 0.26 & 1.7 \\
\hline $\mathrm{Nd}$ & 0.77 & 0.06 & 0.96 & 0.08 & 0.15 & 0.02 & 1.02 & 0.10 & 1.35 & 0.05 & 0.64 & 0.03 & 3.07 & 0.49 & 0.72 & 0.07 & 1.00 & 0.26 & 1.6 \\
\hline $\mathrm{Sm}$ & 0.81 & 0.16 & 1.07 & 0.08 & 0.17 & 0.02 & 1.06 & 0.10 & 1.21 & 0.08 & 0.66 & 0.05 & 2.48 & 0.37 & 0.70 & 0.07 & 1.00 & 0.25 & 1.5 \\
\hline Eu & 0.94 & 0.09 & 1.34 & 0.06 & 0.19 & 0.02 & 0.99 & 0.08 & 1.50 & 0.10 & 0.75 & 0.05 & 2.79 & 0.39 & 0.76 & 0.04 & 1.10 & 0.25 & 1.4 \\
\hline $\mathrm{Gd}$ & 0.85 & 0.16 & 1.08 & 0.08 & 0.16 & 0.02 & 1.00 & 0.06 & 1.18 & 0.06 & 0.70 & 0.05 & 2.61 & 0.40 & 0.74 & 0.08 & 1.07 & 0.28 & 1.3 \\
\hline $\mathrm{Tb}$ & 0.89 & 0.06 & 1.13 & 0.08 & 0.18 & 0.01 & 0.91 & 0.08 & 1.10 & 0.09 & 0.70 & 0.06 & 2.53 & 0.54 & 0.74 & 0.07 & 1.00 & 0.29 & 1.1 \\
\hline Dy & 0.97 & 0.15 & 1.16 & 0.11 & 0.19 & 0.01 & 0.92 & 0.07 & 1.15 & 0.13 & 0.70 & 0.08 & 2.48 & 0.57 & 0.76 & 0.07 & 0.98 & 0.29 & 1.0 \\
\hline Ho & 0.96 & 0.15 & 1.20 & 0.07 & 0.13 & 0.01 & 1.03 & 0.12 & 1.08 & 0.09 & 0.69 & 0.06 & 2.78 & 0.61 & 0.80 & 0.07 & 1.04 & 0.30 & 0.9 \\
\hline Y & 0.78 & 0.09 & 1.00 & 0.03 & 0.15 & 0.02 & 1.05 & 0.15 & 1.14 & 0.15 & 0.73 & 0.09 & 3.18 & 0.66 & 0.83 & 0.08 & 1.10 & 0.33 & 1.0 \\
\hline $\mathrm{Er}$ & 1.01 & 0.18 & 1.24 & 0.10 & 0.21 & 0.01 & 1.20 & 0.29 & 1.11 & 0.10 & 0.72 & 0.06 & 2.84 & 0.68 & 0.81 & 0.08 & 1.01 & 0.29 & 0.8 \\
\hline $\mathrm{Tm}$ & 1.07 & 0.07 & 1.28 & 0.09 & 0.21 & 0.02 & 1.38 & 0.42 & 1.08 & 0.10 & 0.73 & 0.07 & 2.79 & 0.60 & 0.81 & 0.08 & 1.06 & 0.30 & 0.7 \\
\hline $\mathrm{Yb}$ & 1.18 & 0.07 & 1.23 & 0.10 & 0.20 & 0.02 & 1.20 & 0.16 & 1.14 & 0.12 & 0.74 & 0.08 & 2.74 & 0.56 & 0.82 & 0.09 & 1.04 & 0.30 & 0.7 \\
\hline Lu & 1.18 & 0.14 & 1.39 & 0.04 & 0.20 & 0.02 & 1.22 & 0.19 & 1.17 & 0.13 & 0.76 & 0.08 & 2.82 & 0.62 & 0.85 & 0.11 & 1.08 & 0.33 & 0.5 \\
\hline
\end{tabular}

$D_{\text {REE }}$ of calcites, published in our previous work [39], are close to zero: 0.02-0.04 in NCF samples and 0.04-0.1 in sample with NCFP-6. In experiments with the NCFP mixtures, presented in this work, the averages of $D_{\text {REE }}$ are much higher: $0.1-0.2$ and $0.52-1.39$ (Fig. 3a). The averages of $D_{\text {REE }}$ in NCP samples vary in $0.55-1.4$, but taking into account the error for each individual element, it is almost the same (Fig. $3 \mathrm{~b}$ ). In experiments with the highest temperatures the highest $D_{\text {REE }}$ are observed for both mixtures.

NCPSi samples demonstrate highest $D_{\text {REE }}$ with large variations (Fig. 3c). \#7 NCPSi-1 sample demonstrates the highest DREE among them, while $D_{\text {REE }}$ of \#10 NCPSi-2 and \#12 NCPSi-3 is almost equal. This is probably directly related to the content of $\mathrm{SiO}_{2}$ and $\mathrm{P}_{2} \mathrm{O} 5$ of the samples.

The graphs of $D_{\text {REE }}$ of NCPS samples are almost flat, with a slight positive slope. The average $D_{\text {LREE }}$ in \#5 NCPS-1 vary in 1.2-1.7 with strong Eu anomaly, while the coefficients of other elements is about 1.2 and almost equal with \#11 NCPS-2.

\section{Discussion}

\section{Factors influencing the partitioning of REE into calcite in carbonatite systems}

As seen from natural carbonatite samples, the early generations of calcites are characterized by increased content of cations, which size doesn't allow stoichiometrically replace $\mathrm{Ca}^{2+}$ at crustal conditions: $\mathrm{Mg}, \mathrm{Sr}, \mathrm{Ba}$ are most abundant among them $[44,46]$. Sometimes it leads to the formation of Ca-Ba-Sr "protocarbonates", unstable and decaying into calcite and baritocalcite in the form of a solid solution. With the progressive crystallization of calcite, the content of these cations decreases and the trend is replaced by an increased content of Mn and Fe. Consequently, elevated temperatures favor the entry of cations, whose size precludes their unlimited substitution for $\mathrm{Ca}^{2+}$. Along with these cations, REEs can also enter the structure of calcite, which is also noted in natural samples: for example, the correlation between $\mathrm{Ba}$ and $\mathrm{Pb}$ and LREE in early calcites of the Aley complex (Canada) [40,44].

The strong effect of temperature is confirmed by our experiments: in most experiments $D_{\text {REE }}>1$ in high temperatures $\left(900-820^{\circ} \mathrm{C}\right)$, while at other temperatures it's about unity or lower [39].

As follows from a comparison of these experiments and the previous ones [39], the presence of phosphorus is another (and possibly key) positive factor for the fractionation of REE into calcite in carbonatite systems with a high $\mathrm{Na}_{2} \mathrm{O}$ content, but it is difficult to make an unambiguous conclusion between its content and the value of DREE. Another powerful driver for increasing $\mathrm{D}_{\mathrm{REE}}$ is $\mathrm{SiO}_{2}$. Sulfur, on the contrary, promotes the dissolution (retention) of REE in the melt. To evaluate the net effect of these and other parameters (such as $\mathrm{F}, \mathrm{Cl}$ ), additional experiments are required. 


\section{Calcite As A Monitor Of Ree}

Our new data show that at some stage in the evolution of carbonatite magma, with a combination of certain factors, calcite in carbonatites can retain a significant gross REE budget of whole carbonatite and may be a closest proxy for estimation of initial REE content in the melt. For example, at high temperatures, in local areas of a carbonatite body, or when there is a lack of material for fractionation of early traditional mineral concentrators of a large amount of REE (such as apatite, monazite, etc.) even at low concentration of REE in the melt.

Given the high susceptibility of calcite to postmagmatic changes, plasticity and recrystallization, this creates the possibility of releasing from calcite large bulk amounts of REE, Sr and Ba and their subsequent redeposition as a result of interaction with later melts or solutions or as a result of metamorphism. According to various estimates $[40,44]$, such processes can occur at shallow depths already at temperatures of $400-600$ and even $730^{\circ} \mathrm{C}$, which can lead to active loss of REE by calcite, overprinting his textures, and transfer and redeposition of REE far from it due to weakening of the carbonatite body by porous fluids. These events can also be aggravated by recrystallization of calcite during plastic deformation under a stress conditions.

Indeed, patterns of such phenomena occur, for example, in calcites from Afrikanda and Murun carbonatites (Russia) and Bearpaw Mts (USA) [44]: diffusioninduced zoning in primary calcite involving a decrease in $\mathrm{Mn}, \mathrm{Sr}, \mathrm{Ba}$ and REE along grain boundaries and fractures; the $\mathrm{Sr}$ and REE released from calcite are precipitated interstitially as strontianite, baritocalcite, Ba-Sr-Ca-carbonates, barite, burbankite and ancylite.

\section{Stability Of Zircon And Hfse In Carbonatitic Systems}

Gervasoni et al. [45] conducted experiments upon stability of baddeleyite and zircon at $0.7 \mathrm{GPa}$ and $1200^{\circ} \mathrm{C}$ in the system with $\mathrm{CaO} 18-39$ wt. $\%$, $\mathrm{Na}{ }_{2} \mathrm{O} 2-9$ wt.\%, $\mathrm{ZrO}_{2}$ 8-14 wt.\%, MgO 1-2 wt.\%, $\mathrm{SiO}_{2} \mathrm{O}-45 \mathrm{wt} . \%$ and $\mathrm{H}_{2} \mathrm{O} 1-2 \mathrm{wt} . \%$. In these experiments, zircon appears at $\left(\mathrm{CaO}+\mathrm{MgO}^{\left.-\mathrm{Na}_{2} \mathrm{O}\right) / \mathrm{SiO}} \mathrm{O}_{2}=0.5-1.4 \mathrm{in}\right.$ the system. At 2.8-11.1, only baddeleyite is stable. In our experiments, zircon formed at shallower conditions, lower amounts of $\mathrm{ZrO}_{2}$ and $\mathrm{HfO}$, and lower activity of silica: $\left(\mathrm{Ca}+\mathrm{Na}_{2} \mathrm{O}\right) / \mathrm{SiO}_{2}=13.24$ and 22.45 .

Gervasoni et al. [45] also consider the issue of the stability of zircons in carbonatite melts. They conducted an experiment on the solubility of zircon in haplocarbonatite melts with 0 and $17 \mathrm{wt} . \% \mathrm{SiO}_{2}$. In the experiment without $\mathrm{SiO}_{2}$, zircon decomposed into a siliceous melt and baddeleyite, and at $17 \mathrm{wt} \%$ it slightly melted and recrystallized. Based on their experiments, Gervasoni et al. concludes that zircons in carbonatite magmas with $\mathrm{SiO}_{2}<20$ wt.\% cannot survive. Therefore, one should be careful with the dating of zircons from carbonatites, since they may be xenogenic and do not reflect the real time of intrusion of carbonatite magma.

It seems to us that the range between 0 and $17 \mathrm{wt} . \%$ of is quite drastic $\mathrm{SiO}_{2}$, especially since a small amount of silica is almost always presented in natural carbonatites. From our experiments, it follows that zircons can crystallize from carbonatite magmas even with small proportions of $\mathrm{SiO}_{2}$ to other components and in shallower environments.

\section{Methods}

Experimental mixtures were composed of reagent-grade $\mathrm{CaCO}_{3}, \mathrm{Na}_{2} \mathrm{CO}_{3}, \mathrm{Ca}_{3}\left(\mathrm{PO}_{4}\right)_{2}, \mathrm{CaF}_{2}, \mathrm{Na}_{2} \mathrm{Si}_{2} \mathrm{O}_{5}$ and $\mathrm{Na}_{2} \mathrm{SO}_{4}$ and doped with mixtures of trace elements: $\mathrm{Zr}, \mathrm{Hf}, \mathrm{Nb}, \mathrm{Ta}, \mathrm{Ti}, \mathrm{Sr}, \mathrm{W}, \mathrm{Mo}$ (HFSE mixture) and all REE, including Sr and Y (REE mixture) (Table 1). Both mixtures of trace elements were composed of equal weight proportions of reagent-grade $\mathrm{CeF}_{2}, \mathrm{SrCO}_{3}$ and pure oxides of other individual elements. Pure reagents were dried at $180^{\circ} \mathrm{C}$ overnight, mixed under acetone in agate mortar and dried again. The mixtures of NCFP type are direct analogues of our mixtures, used in previous work [39]. About 45-55 mg of the starting mixtures were put in gold capsules and sealed by arc-welding in the flow of Ar. 
Table 1

Nominal major and trace compositions of starting mixtures (in wt\%).

\begin{tabular}{|c|c|c|c|c|c|c|c|c|c|c|}
\hline Components, wt.\% & NCFP-7 & NCFP-8 & NCFP-9 & NCP-1 & NCP-2 & NCPSi-1 & NCPSi-2 & NCPSi-3 & NCPS-1 & NCPS-2 \\
\hline $\mathrm{SiO}_{2}$ & - & - & - & - & - & 4.84 & 2.90 & 2.90 & - & - \\
\hline $\mathrm{CaO}$ & 40.72 & 47.31 & 53.14 & 47.14 & 45.55 & 42.43 & 42.82 & 42.82 & 45.06 & 43.53 \\
\hline $\mathrm{Na}_{2} \mathrm{O}$ & 18.57 & 11.37 & 5.78 & 20.31 & 21.64 & 21.68 & 22.38 & 21.89 & 21.34 & 22.61 \\
\hline $\mathrm{F}$ & 10.12 & 9.19 & 10.62 & 0.03 & 0.01 & 0.02 & 0.01 & 0.01 & 0.02 & 0.01 \\
\hline $\mathrm{Cl}$ & - & - & - & 0.02 & 0.01 & 0.02 & 0.01 & 0.01 & 0.02 & 0.01 \\
\hline $\mathrm{SO}_{3}$ & - & - & - & - & - & - & - & - & 2.50 & 2.50 \\
\hline $\mathrm{P}_{2} \mathrm{O}_{5}$ & 9.15 & 9.15 & 9.15 & 5.33 & 2.65 & 4.80 & 2.49 & 2.49 & 5.10 & 2.54 \\
\hline REE & 0.40 & 0.40 & 0.40 & 0.50 & 0.05 & 0.45 & 0.05 & 0.47 & 0.48 & 0.05 \\
\hline HFSE & 0.40 & 0.40 & 0.40 & 0.03 & 0.05 & 0.02 & 0.05 & 0.05 & 0.02 & 0.05 \\
\hline $\mathrm{CO}_{2}$ & 20.65 & 22.18 & 20.51 & 26.64 & 30.03 & 25.73 & 29.29 & 29.36 & 25.46 & 28.70 \\
\hline Total & 100.00 & 100.00 & 100.00 & 100.00 & 100.00 & 100.00 & 100.00 & 100.00 & 100.00 & 100.00 \\
\hline$\left(\mathrm{CaO}+\mathrm{Na}_{2} \mathrm{O}\right) / \mathrm{SiO}_{2}$ & & & & & & 13.24 & 22.45 & 22.28 & & \\
\hline $\mathrm{CaO} / \mathrm{SiO}$ & & & & & & 8.77 & 14.74 & 14.74 & & \\
\hline
\end{tabular}

Experiments were performed at pressure of $100 \mathrm{MPa}$ and over temperature interval of $650-900^{\circ} \mathrm{C}$ in rapid-quench cold-seal pressure vessels at the German Research Centre for Geosciences (GFZ Potsdam). A detailed description of this type of the vessels described in [41]. The autoclaves at GFZ Potsdam are made of the Ni-Cr alloy Vakumelt ATS 290-G (ThyssenKrupp AG). Oxygen fugacity was not controlled, but believed to have been close to that of the Ni-NiO equilibrium, buffered by oxidation reactions of water, used as pressure medium, and the $\mathrm{Ni}-\mathrm{Cr}$ alloy of the autoclave. Temperature measured by external $\mathrm{Ni}-$ $\mathrm{CrNi}$ thermocouple, calibrated against the melting temperature of gold. Temperature measurements are corrected for a temperature gradient inside the autoclaves, which was measured using a second inner thermocouple during the initial calibration of the vessels. The external thermocouple has an error of approximately $\pm 1^{\circ} \mathrm{C}$, and the total error of temperature measurements, including the uncertainties due to the temperature gradients, is estimated to be $\pm 5^{\circ} \mathrm{C}$. Pressure is measured by transducers, and results were checked against a pressure gauge. The transducers and gauge are factory calibrated and have an accuracy of better than $\pm 0.1 \mathrm{MPa}$. Run times varied from 20 to 141 hours. In experiments with decreasing temperature, the temperature lowered gradually, with decrease of $5-7^{\circ} \mathrm{C}$ in every $1-1.5$ hour. Samples were kept for at least 10 hours at the final temperature before quenching. The quenching of the experimental samples in such apparatus is isobaric and less than a second.

After the experiments, samples were mounted in epoxy resin and polished with diamond polishing pastes without water to avoid dissolution of alkalis. Major components of the run products were analyzed using Cameca SX-50 and SX-100 electron microprobes in the GFZ Potsdam and energy-dispersive spectrometry (EDS) in combination with back-scattered electron imaging (BSE) using a MIRA 3 LMU SEM (TESCAN Ltd.) equipped with an INCA Energy 450 XMax 80 microanalysis system (Oxford Instruments Ltd.) in the Analytical Center for multi-elemental and isotope research SB RAS (Novosibirsk, Russia).

Microprobe analyses were performed in WDS mode with a $10 \mathrm{nA}$ beam current and accelerating voltage of $15 \mathrm{kV}$. Counting time for F (analyzed with TAP crystal) was $40 \mathrm{~s}$. For all other elements it was set to $20 \mathrm{~s}$ on peak and $10 \mathrm{~s}$ on background. These parameters are benign to avoid any damage to carbonates or melt. The following synthetic and natural standards were used for the calibration: wollastonite (Ca), albite and jadeite (Na), apatite (P), LiF ( $F)$. Quenched melts were analyzed with a defocused beam (beam diameter of 20-40 $\mu \mathrm{m}$ ). At least five point analyses were performed on each phase to obtain statistically representative averages.

For EDS analyses the samples were coated with a $25 \mu \mathrm{m}$ conductive carbon coating. EDS analyses were made at low vacuum of $60-80 \mathrm{~Pa}$, an accelerating voltage of $20 \mathrm{kV}$, a probe current of $1 \mathrm{nA}$, and accumulation time of $20 \mathrm{~s}$. The simple compounds and metals were used as reference samples for most of the elements: $\mathrm{SiO}_{2}$ (Si-Ka and O-Ka), diopside (Ca-Ka), albite (Na-Ka), $\mathrm{Ca}_{2} \mathrm{P}_{2} \mathrm{O}_{7}(\mathrm{P}-\mathrm{Ka}), \mathrm{BaF}_{2}$ (F-Ka), pyrite (S-Ka), SrF 2 (Sr-Ka) and various (LREE)PO 4 (LREE-La). Correction for matrix effects was made by the XPP algorithm, implemented in the software of the microanalysis system. Metallic Co served for quantitative optimization (normalization to probe current and energy calibration of the spectrometer).

Trace elements were measured by LA-ICP-QQQ-MS applying a Geolas Compex Pro $193 \mathrm{~nm}$ excimer laser coupled to a Thermo iCAP TQ mass spectrometer in the GFZ Potsdam. Analytical conditions comprised a spot size of $24 \mu \mathrm{m}$, repetition rate of $8 \mathrm{~Hz}$, and laser energy density of $5 \mathrm{~J} \mathrm{~cm}^{-2}$. NIST SRM $610 \mathrm{was}$ used as external standard and Ca as derived from EPMA as internal standard. BCR-2G was analyzed as known-unknown for accuracy control and trace element concentrations are in agreement with published data at $<10 \% 2 \sigma$ RSD. Each analysis comprised $20 \mathrm{~s}$ background, $40 \mathrm{~s}$ ablation and $20 \mathrm{~s}$ wash-out. Data was processed using the trace elements IS data reduction scheme [42] in iolite 3.63 [43].

\section{Declarations}


Experiments, microprobe and LA-ICP-MS analyzes supported by Russian Science Foundation (19-17-00013). The EDS analyses done on state assignment of IGM SB RAS (0330-2019-0002).

\section{Author contributions}

D.A.C. developed the idea of the paper, conducted the experiments, collected and interpreted the analytical data, wrote and designed the original manuscript; C.W.-U. collected the analytical data and reviewed the original manuscript.

\section{Competing interests}

The author declare no competing interests

\section{References}

1. Woolley, A. R. \& Kjarsgaard, B. A. Carbonatite occurrences of the world: map and database. Geol. Surv. of Canada. 28 pages (1 sheet), 1 CD-ROM, doi:10.4095/225115 (2008).

2. Verplanck, P. L., Mariano, A. N. \& Mariano, A. Rare earth ore geology of carbonatites. In: Verplanck, P.L., Hitzman, M.W. (Eds.). Rare Earth and Critical Elements in Ore Deposits. Soc. of Ec. Geol., Littleton, 5-32(2016).

3. Woolley, A. R. \& Kjarsgaard, B. A. Paragentic types of carbonatite as indicated by the diversity and relative abundances of associated silica rocks: evidence from a global database. Can. Mineral, 46, 741-752 (2008).

4. Wyllie, P. J. \& Huang, W. L. Carbonation and melting reactions in the system CaO-MgO-SiO2-CO2 at mantle pressures with geophysical and petrological applitations. Contrib. Mineral. Petrol, 54, 79-107 (2008).

5. Wallace, M. E. \& Green, D. H. An experimental determination of primary carbonatite magma composition. Nature, 335, 343-346 (1988).

6. Falloon, T. J. \& Green, D. H. The solidus of carbonated, fertile peridotite. Earth Planet. Sci. Let, 94, 364-370 (1989).

7. Falloon, T. J. \& Green, D. H. The solidus of carbonated fertile peridotite under fluid-saturated conditions. Geology, 18, 195-199 (1990).

8. Dalton, J. A. \& Presnall, D. C. Carbonatitic melts along the solidus of model Iherzolite in the system CaO-MgO-Al2O3-SiO2-CO2 from 3 to 7 GPa. Contrib. Min. Petr, 131, 123-135 (1998).

9. Dalton, J. A. \& Presnall, D. C. The continuum of primary carbonatitic-kimberlitic melt compositions in equilibrium with Iherzolite: data from the system CaO-MgO-Al2O3-SiO2-CO2 at 6 GPa. J. Petrol, 39, 1953-1964 (1998).

10. Gudfinnsson, G. H. \& Presnall, D. C. Continuous gradation among primary carbonatitic, kimberlitic, melilititic, basaltic, picritic, and komatiitic melts in equilibrium with garnet Iherzolite at 3-8 GPa. J. Petrol, 46, 1645-1659 (2005).

11. Dasgupta, R. \& Hirschmann, M. M. Melting in the Earth's deep upper mantle caused by carbon dioxide. Nature, 440, 659-662 (2006).

12. Dasgupta, R. \& Hirschmann, M. M. Effect of variable carbonate concentration on the solidus of mantle peridotite. Am. Min, 92, 370-379 (2007).

13. Brey, G. P., Bulatov, V. K., Girnis, A. V. \& Lahaye, Y. Experimental melting of carbonated peridotite at 6-10 GPa. J. Petrol, 49, 797-821 (2008).

14. Foley, S. F. et al. The composition of near-solidus melts of peridotite in the presence of $\mathrm{CO} 2$ and $\mathrm{H} 2 \mathrm{O}$ between 40 and $60 \mathrm{kbar}$. Lithos, 112, 274-283 (2009).

15. Brey, G. P., Bulatov, V. K. \& Girnis, A. V. Melting of K-rich carbonated peridotite at 6-10 GPa and the stability of K-phases in the upper mantle. Chem. Geol, 281, 333-342 https://doi.org/10.1016/j.chemgeo.2010.12.019 (2011).

16. Kjarsgaard, B. A. \& Hamilton, D. L. The genesis of carbonatites by immiscibility. In Carbonatites: Genesis and Evolution. London. 388-404(1989).

17. Lee, W. \& Wyllie, P. J. Experimental data bearing on liquid immiscibility, crystal fractionation and the origin of calciocarbonatites and natrocarbonatites. Int. Geol. Rev, 36, 797-819 (1994).

18. Otto, J. W. \& Wyllie, P. J. Relationship between silicate melts and carbonate-precipitating melts in $\mathrm{CaO}-\mathrm{MgO}-\mathrm{SiO} 2-\mathrm{CO} 2-\mathrm{H} 2 \mathrm{O}$ at 2 kbar. Miner. Petrol, 48, 343-365 (1993).

19. Veksler, I. V., Nielsen, T. F. D. \& Sokolov, S. V. Mineralogy of crystallized melt inclusions from Gardiner and Kovdor ultramafic alkaline complexes: implications for carbonatite genesis. J. Petrol, 39, 2015-2031 (1998).

20. Le Bas, M. J. Diversification of carbonatite. In: Bell, K. (Ed.), Carbonatites: Genesis and Evolution. Unwin Hyman, London. 428-445(1989).

21. Xu, C. et al. Flat rare earth element patterns as an indicator of cumulate processes in the Lesser Qinling carbonatites, China. Lithos, 95, 267-278 (2007).

22. Chakhmouradian, A. R., Mumin, A. H., Demeny, A. \& Elliott, B. Postorogenic carbonatites at Eden Lake, Trans-Hudson Orogen (northern Manitoba, Canada). Lithos, 103, 503-526 (2008).

23. Veksler, I. V., Petibon, C., Jenner, G., Dorfman, A. M. \& Dingwell, D. B. Trace element partitioning in immiscible silicate and carbonate liquid systems: an initial experimental study using a centrifuge autoclave. J. Petrol, 39, 2095-2104 (1998).

24. Veksler, I. V. et al. Partitioning of elements between silicate melt and immiscible fluoride, chloride, carbonate, phosphate and sulfate melts with implications to the origin of natrocarbonatite. Geochim. Cosmochim. Acta, 79, 20-40 (2012).

25. Dasgupta, R., Hirschmann, M. M., McDonough, W. F., Spiegelman, M. \& Withers, A. C. Trace element partitioning between garnet Iherzolite and carbonatite at 6.6 and $8.6 \mathrm{GPa}$ with applications to the geochemistry of the mantle and of mantle-derived melts, Chem. Geol.262,57-77, https://doi.org/10.1016/j.chemgeo.2009.02.004 (2009). 
26. Jones, J. H., Walker, D., Picket, D. A., Murrel, M. T. \& Beate, P. Experimental investigations of the partitioning of Nb, Mo, Ba, Ce, Pb, Ra, Th, Pa and U between immiscible carbonate and silicate liquids. Geochim. Cosmochim. Acta, 59, 1307-1320 (1995).

27. Prowatke, S. \& Klemme, S. Rare earth element partitioning between titanite and silicate melts: Henry's law revisited. Geochim. Cosmochim. Acta, 70, 4997-5012 (2006).

28. Blundy, J. \& Dalton, J. Experimental comparison of trace element partitioning between clinopyroxene and melt in carbonate and silicate systems, and implications for mantle metasomatism. Contrib. Min. Petrol, 139, 356-371 (2000).

29. Ryabchikov, I. D., Orlova, G. P., Senin, V. G. \& Trubkin, N. V. Partitioning of rare earth elements between phosphate-rich carbonatite melts and mantle peridotites. Mineral. Petrol, 49, 1-12 (1993).

30. Girnis, A. V., Bulatov, V. K., Brey, G. P., Gerdes, A. \& Höfer, H. E. Trace element partitioning between mantle minerals and silico-carbonate melts at 6-12 GPa and applications to mantle metasomatism and kimberlite genesis. Lithos, 160-161, 183-200 (2013).

31. Klemme, S., van der Laan, S. R., Foley, S. F. \& Günther, D. Experimentally determined trace and minor element partitioning between clinopyroxene and carbonatite melt under upper mantle conditions. Earth Planet. Sci. Let, 133, 439-448 (1995).

32. Gaetani, G. A., Kent, A. J. R., Grove, T. L., Hutcheon, I. D. \& Stolper, E. M. Mineral/melt partitioning of trace elements during hydrous peridotite partial melting. Contrib. Min. Petrol, 145, 391-405 (2003).

33. Green, T. H., Adam, J. \& Sie, S. H. Trace element partitioning between silicate minerals and carbonatite at 25 kbar and application to mantle metasomatism. Min. Petrol, 46, 179-184 (1992).

34. Green, T. H. Experimental studies of trace-element partitioning applicable to igneous petrogenesis m Sedona 16 years later. Chem. Geol, 117, 1-36 (1994).

35. Sweeney, R. J., Prozesky, V. \& Przybylowicz, W. Selected trace and minor element partitioning between peridotite minerals and carbonatite melts at $18-46$ kbar pressure. Geochim. Cosmochim. Acta, 59, 3671-3683 (1995).

36. Hammouda, T., Moine, B. N., Devidal, J. L. \& Vincent, C. Trace element partitioning during partial melting of carbonated eclogites. Phys. Earth Planet. Inter, 174, 60-69 (2009).

37. Hammouda, T., Chantel, J. \& Devidal, J. L. Apatite solubility in carbonatitic liquids and trace element partitioning between apatite and carbonatite at high pressure. Geochim. Cosmochim. Acta, 74, 7220-7235 (2010).

38. Le Maitre, R. W. Igneous rocks: a classification and glossary of terms: recommendations of International Union of Geological Sciences Subcommission on the Systematics of Igneous Rocks.Cam. Un. Pr.236 (2002).

39. Chebotarev, D. A., Veksler, I. V., Wohlgemuth-Ueberwasser, C., Doroshkevich, A. G. \& Koch-Müller, M. Experimental study of trace element distribution between calcite, fluorite and carbonatitic melt in the system CaCO3 + CaF2 + Na2CO3 \pm Ca3(PO4)2 at 100 MPa. Contrib. Min. Petrol, 174, 4 (2019).

40. Chakhmouradian, A. R., Reguir, E. P. \& Couëslan, C. Calcite and dolomite in intrusive carbonatites. II. Trace-element variations. Min. Petrol, 110, $361-377$ https://doi.org/10.1007/s00710-015-0392-4 (2016).

41. Matthews, W., Linnen, R. L. \& Guo, Q. A filler-rod technique for controlling redox conditions in cold-seal pressure vessels. Am. Min, 88, 701-707 (2003).

42. Woodhead, J., Hellstrom, J., Hergt, J., Greig, A. \& Maas, R. Isotopic and elemental imaging of geological materials by laser ablation Inductively Coupled Plasma mass spectrometry. J. Geostand. Geoanal. Res, 31, 331-343 (2007).

43. Paton, C. et al. Freeware for the visualisation and processing of mass spectrometric data. J. Analyt. Atom. Spectrom, https://doi.org/10.1039/c1ja10172b (2011).

44. Chakhmouradian, A. R., Reguir, E. P. \& Zaitsev, A. N. Calcite and dolomite in intrusive carbonatites. I. Textural variations. Min. Petrol, 110, 333-360 https://doi.org/10.1007/s00710-015-0390-6 (2016).

45. Gervasoni, F., Klemme, S., Rohrbach, A., Grutzner, T. \& Berndt, J. Experimental constraints on the stability of baddeleyite and zircon in carbonate- and silicate-carbonate melts. Am. Min, 102, 860-866 (2017).

46. Saito, A. et al. Incorporation of Incompatible Strontium and Barium lons into Calcite (CaCO3) through Amorphous Calcium Carbonate. Minerals, 10, 270 https://doi.org/10.3390/min10030270 (2020).

\section{Figures}



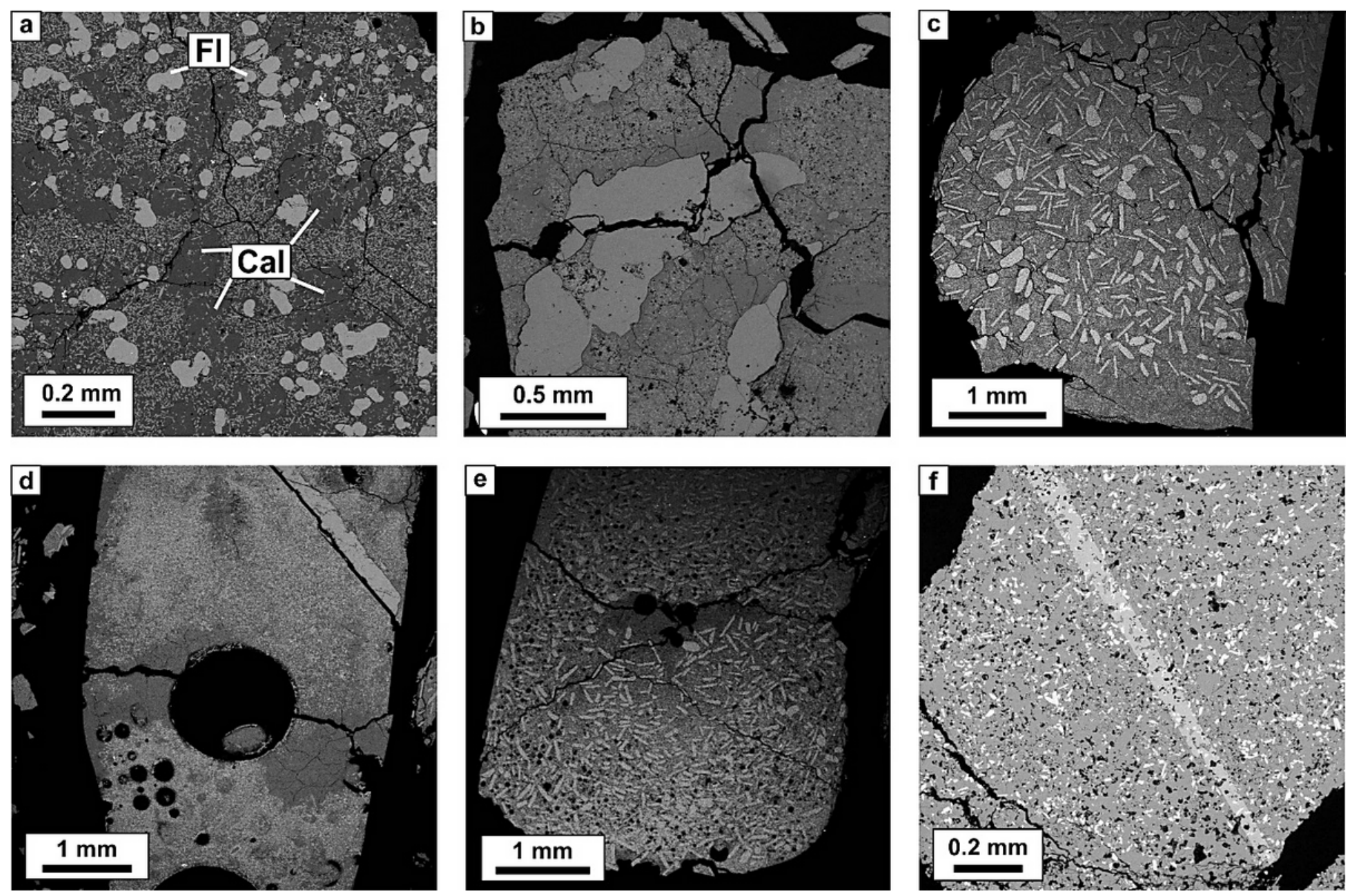

Figure 1

Morphology of calcites formed in experiments: (a) \#3 NCFP-9; (b) \#4 NCP-1; (c) \#6 NCP-1; (d) \#7 NCPSi-1; (e) \#8 NCPS-1; (f) \#10 NCPSi-3. 

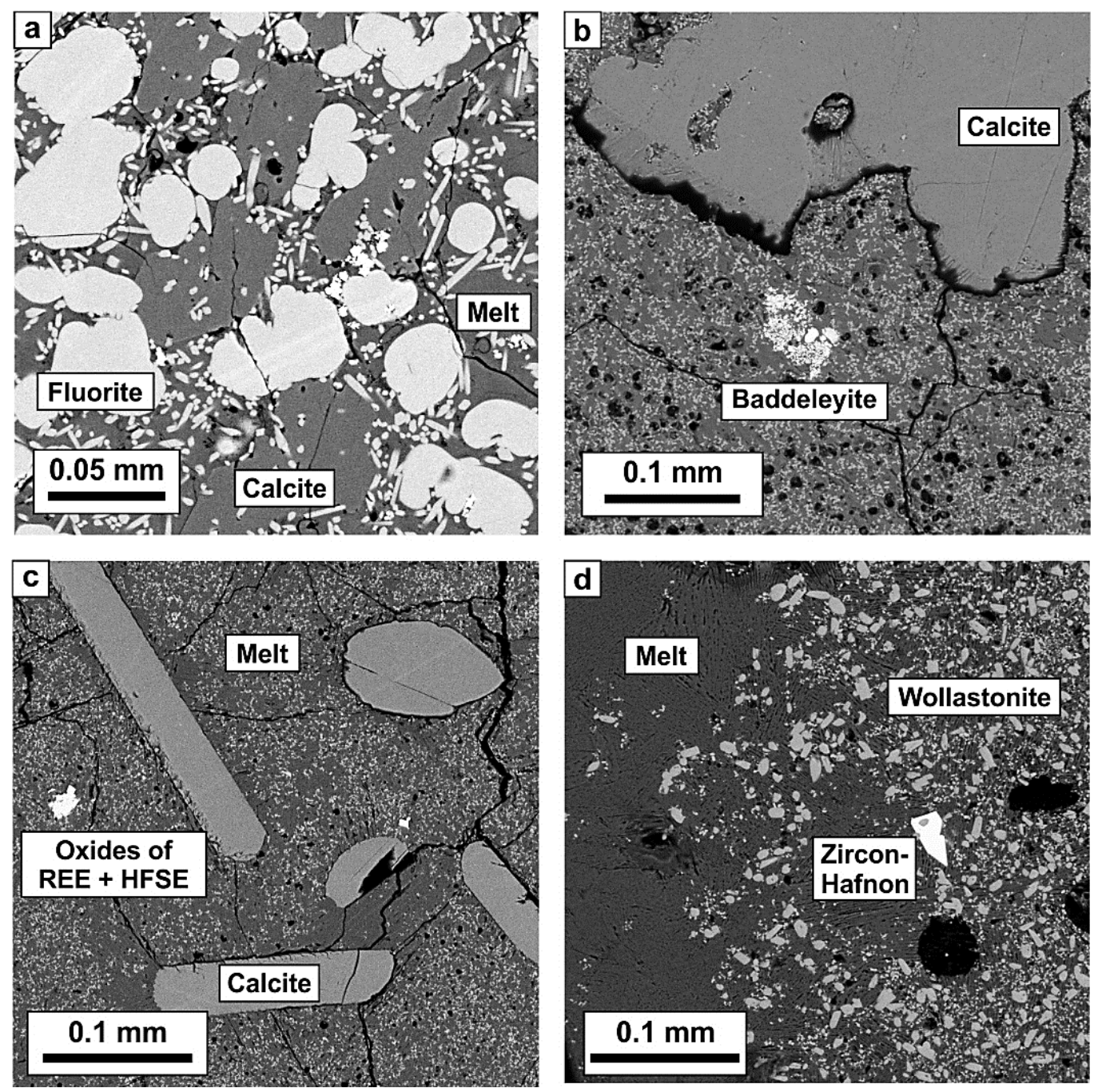

Figure 2

Textures of the experimental samples: (a) \#3 NCFP-9; (b) \#4 NCP-1; (c) \#6 NCP-1; (d) \#7 NCPSi-1. 

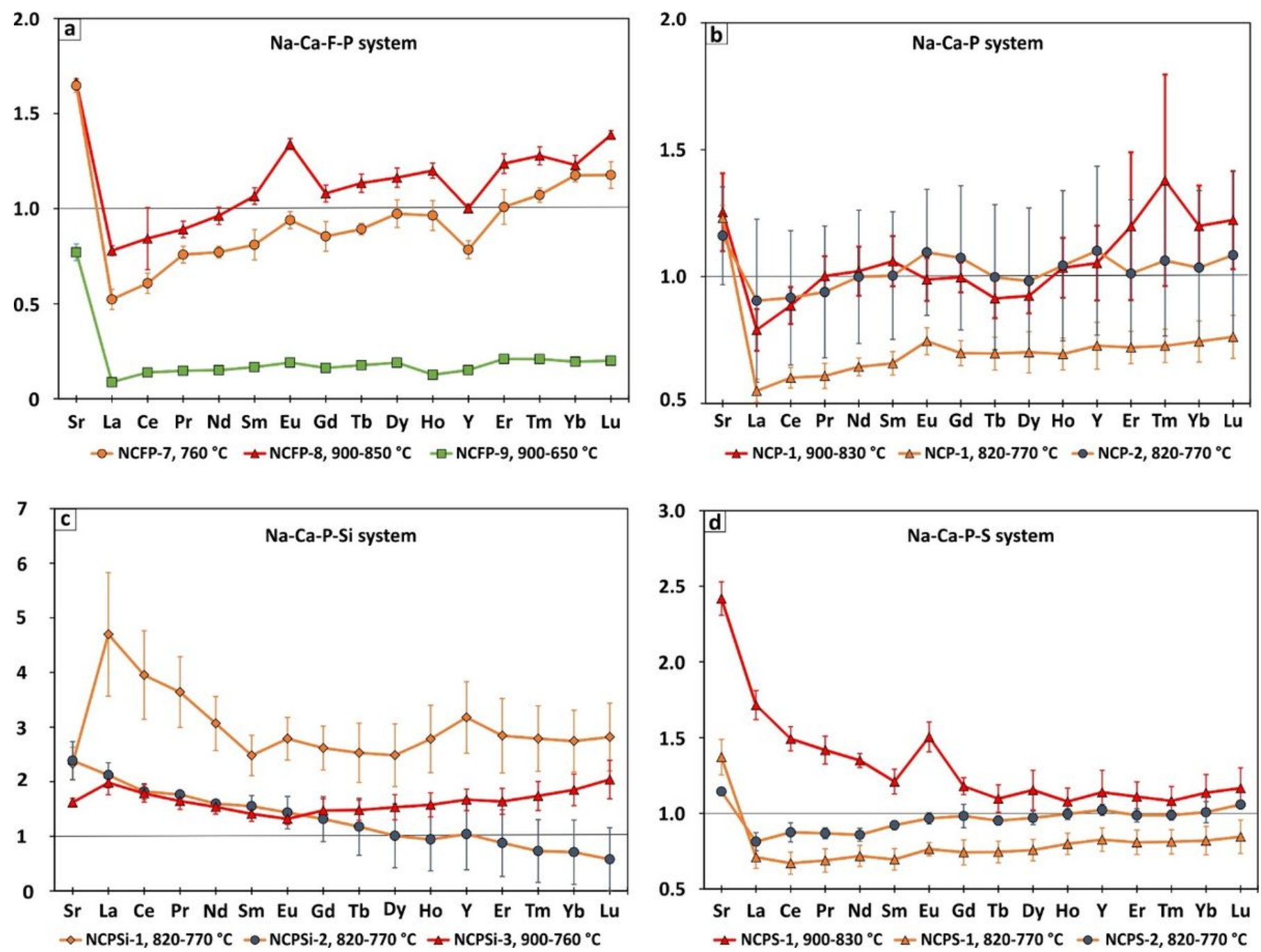

Figure 3

The average partition coefficients of REE with the standard deviation as error bars for calcites. 